\title{
Some notes on complex symmetric operators
}

\author{
Marcos S. Ferreira \\ Communicated by Sergey Astashkin
}

\begin{abstract}
In this paper we show that every conjugation $C$ on the Hardy-Hilbert space $H^{2}$ is of type $C=T^{*} \mathcal{J} T$, where $T$ is an unitary operator and $\mathcal{J} f(z)=\overline{f(\bar{z})}$ with $f \in H^{2}$. Moreover we prove some relations of complex symmetry between the operators $T$ and $|T|$, where $T=U|T|$ is the polar decomposition of bounded operator $T \in \mathcal{L}(\mathcal{H})$ on the separable Hilbert space $\mathcal{H}$.
\end{abstract}

Keywords. Hardy space, Toeplitz operator, complex symmetric operator, Aluthge transform.

2020 Mathematics Subject Classification. 46C99, 47B99.

\section{Introduction}

Let $\mathcal{L}(\mathcal{H})$ be the space of bounded linear operators on a separable Hilbert space $\mathcal{H}$. A conjugation $C$ on $\mathcal{H}$ is an antilinear operator $C: \mathcal{H} \rightarrow \mathcal{H}$ such that $C^{2}=I$ and $\langle C f, C g\rangle=\langle g, f\rangle$, for all $f, g \in \mathcal{H}$. An operator $T \in \mathcal{L}(H)$ is said to be complex symmetric if there exists a conjugation $C$ on $H$ such that $C T=T^{*} C$ (we will often say that $T$ is $C$-symmetric). Complex symmetric operators generalize the concept of symmetric matrices of linear algebra. Indeed, it is well known ([5, Lemma 1]) that given a conjugation $C$, there exists an orthonormal basis $\left\{f_{n}\right\}_{n=0}^{\infty}$ for $\mathcal{H}$ such that $C f_{n}=f_{n}$. Hence, if $T$ is $C$-symmetric then

$$
\left\langle T f_{n}, f_{m}\right\rangle=\left\langle C f_{m}, C T f_{n}\right\rangle=\left\langle f_{m}, T^{*} C f_{n}\right\rangle=\left\langle T f_{m}, f_{n}\right\rangle
$$

that is, $T$ has a symmetric matrix representation. The converse result is also true. That is, if there is an orthonormal basis such that $T$ has a symmetric matrix representation, then $T$ is complex symmetric.

The complex symmetric operators class was initially addressed by Garcia and Putinar [5,6] and includes the normal operators, Hankel operators and Volterra integration operators.

Now, let $L^{2}$ be the Hilbert space on the unit circle $\mathbb{T}$ and let $L^{\infty}$ be the Banach space of all essentially bounded functions on $\mathbb{T}$. It is known that $\left\{e^{i n \theta}: n \in \mathbb{Z}\right\}$ 
is an orthonormal basis for $L^{2}$. The Hardy-Hilbert space, denoted by $H^{2}$, consists of all analytic functions $f(z)=\sum_{n=0}^{\infty} a_{n} z^{n}$ on the unit disk $\mathbb{D}$ such that $\sum_{n=0}^{\infty}\left|a_{n}\right|^{2}<\infty$. It is clear that $\left\{z^{n}: n=0,1,2, \ldots\right\}$ is an orthonormal basis for $H^{2}$.

For each $\phi \in L^{\infty}$, the Toeplitz operator $T_{\phi}: H^{2} \rightarrow H^{2}$ is defined by

$$
T_{\phi} f=P(\phi f),
$$

for each $f \in H^{2}$, where $P: L^{2} \rightarrow H^{2}$ is the orthogonal projection. The concept of Toeplitz operators was initiated by Brown and Halmos [1] and generalizes the concept of Toeplitz matrices.

In [7], Guo and Zhu raised the question of characterizing complex symmetric Toeplitz operators on $H^{2}$ in the unit disk. In order to obtain such characterization, Ko and Lee [8] introduced the family of conjugations $C_{\lambda}: H^{2} \rightarrow H^{2}$, given by

$$
C_{\lambda} f(z)=\overline{f(\lambda \bar{z})}
$$

with $\lambda \in \mathbb{T}$ and proved the following result:

Theorem 1.1. Let $\phi(z)=\sum_{n=-\infty}^{\infty} \widehat{\phi}(n) z^{n} \in L^{\infty}$. Then $T_{\phi}$ is $C_{\lambda}$-symmetric if, and only if, $\widehat{\phi}(-n)=\lambda^{n} \widehat{\phi}(n)$, for all $n \in \mathbb{Z}$.

\section{Canonical conjugations}

Our first objective in this paper is to study relations between an arbitrary conjugation $C$ on $H^{2}$ and the conjugation $\mathcal{J} f(z)=\overline{f(\bar{z})}$. Once the conjugation $\mathcal{J}$ is a kind of canonical conjugation on $H^{2}$, we observe a close relationship between conjugations of $H^{2}$ and conjugation $\mathcal{J}$, namely:

Theorem 2.1. If $C$ is an conjugation on $H^{2}$, then exists an unitary operator $T$ : $H^{2} \rightarrow H^{2}$ such that $T C=\mathcal{J} T$.

Proof. Since $C$ is an conjugation, there exists an orthonormal basis $\left\{f_{n}\right\}_{n=0}^{\infty}$ of $H^{2}$ such that $C f_{n}=f_{n}$. Now, let $\left\{z^{n}\right\}_{n=0}^{\infty}$ the standard orthonormal basis of $H^{2}$ and the linear isomorphism $T: H^{2} \rightarrow H^{2}$ given by

$$
T\left(\sum_{n=0}^{\infty} a_{n} f_{n}\right)=\sum_{n=0}^{\infty} a_{n} z^{n} .
$$


Note that $T f_{n}=z^{n}$, for all $n \geq 0$, and therefore $T$ is unitary. Now, for $f(z)=\sum_{n=0}^{\infty} a_{n} z^{n} \in H^{2}$, we get

$$
\begin{aligned}
\mathcal{J} f(z) & =\sum_{n=0}^{\infty} \overline{a_{n}} T\left(f_{n}\right) \\
& =T\left(\sum_{n=0}^{\infty} \overline{a_{n}} C f_{n}\right) \\
& =\left(T C T^{-1}\right) f(z),
\end{aligned}
$$

whence $\mathcal{J} T=T C$.

The previous theorem says that all complex symmetric Toeplitz operator is unitarily equivalent to a $\mathcal{J}$-symmetric operator. Indeed, we have:

Remark 2.2. Let $T_{\phi}: H^{2} \rightarrow H^{2}$ an Toeplitz operator. Observe that, if $T_{\phi}$ is $C$-symmetric, since the operator $T$ of previous theorem is unitary, we have

$$
\mathcal{J}=T C T^{*},
$$

therefore the operator $T_{2}=T T_{\phi} T^{*}$ is $\mathcal{J}$-symmetric (see [5, p. 1291]). This shows that $T_{\phi}$ and $T_{2}$ are unitarily equivalent operators. Moreover, is obvious that, if $T$ commutes with $\mathcal{J}$ or $C$, then $C=\mathcal{J}$.

The converse of the Theorem 2.1 is true and your proof is immediate.

Proposition 2.3. If $T: H^{2} \rightarrow H^{2}$ is an unitary operator, then $C=T^{-1} \mathcal{J} T$ is an conjugation on $H^{2}$.

In short, the Theorem 2.1 and Proposition 2.3 tell us that:

Corollary 2.4. If $T: H^{2} \rightarrow H^{2}$ an linear isomorphism and $C=T^{-1} \mathcal{J} T$, then $T$ is unitary if, and only if, $C$ is a conjugation on $H^{2}$.

We already know that every normal operator is complex symmetric and that the reciprocal in general is not true. However, for Toeplitz operators, by Theorem 1.1 we have that if $T_{\phi}$ is $\mathcal{J}$-symmetric, then $T_{\phi}$ is normal. Now note that if $T_{\phi}$ is normal not necessarily $T_{\phi}$ is $\mathcal{J}$-symmetric. In fact, if $\phi(z)=-\bar{z}+z$ then $T_{\phi}$ is normal, however is not $\mathcal{J}$-symmetric.

Matrix of operators $\mathcal{J}$-symmetric on $H^{2}$ are quite simple to determine. The proof of the next result is left to the reader.

Proposition 2.5. Let $A \in \mathcal{L}\left(H^{2}\right)$. Then $A$ is $\mathcal{J}$-symmetric if, and only if, the matrix of $A$ with respect the canonical basis of $H^{2}$ is symmetric. 


\section{Properties of complex symmetry}

In the following, we present some properties of complex symmetry in Hilbert spaces. The first result gives us a way to get complex symmetric operators from another complex symmetric operator. First, we need some lemmas:

Lemma 3.1. ([6, Lemma 1]) If $C$ and $J$ are conjugations on a Hilbert space $\mathcal{H}$, then $U=C J$ is a unitary operator. Moreover, $U$ is both $C$-symmetric and $J$ symmetric.

Lemma 3.2. ([3, Lemma 2.2]) If $U: \mathcal{H} \rightarrow \mathcal{H}$ is a unitary and complex symmetric operator with conjugation $C$, then $U C$ is a conjugation.

Proposition 3.3. Let $T: \mathcal{H} \rightarrow \mathcal{H}$ an operator and $C$ and $J$ conjugations on $\mathcal{H}$. Then $T$ is $C$-symmetric if, and only if, $U T$ is $U C$-symmetric, where $U=C J$.

Proof. We already know that $U$ is unitary and $C$ and $J$-symmetric and that $U C=$ $C J C$ is a conjugation, by Lemmas 3.1 and 3.2. Now since $U^{*}=U^{-1}=J C$ and $T$ is $C$-symmetric, we have

$$
U T(U C)=U T C U^{*}=U C T^{*} U^{*}=U C(U T)^{*} .
$$

Reciprocally, suppose that $U C(U T)^{*}=U T(U C)$. Thus

$$
\begin{aligned}
C T^{*} U^{*} & =C(U T)^{*} \\
& =U^{*} U C(U T)^{*} \\
& =U^{*} U T U C \\
& =T U C \\
& =T C U^{*},
\end{aligned}
$$

whence $C T^{*}=T C$.

Lemma 3.4. If $T: \mathcal{H} \rightarrow \mathcal{H}$ is both $C$-symmetric and $J$-symmetric, then $T$ is both $C J C$-symmetric and $J C J$-symmetric.

Proof. By Lemma 3.1, we have that $U:=C J$ is unitary and $C$ and $J$-symmetric. Hence, by Lemma 3.2, $U C=C J C$ is a conjugation on $\mathcal{H}$. Thus, since $C T=$ $T^{*} C$ and $J T=T^{*} J$ we get

$$
(C J C) T=C(T J) C=T^{*}(C J C),
$$

and so $T$ is $C J C$-symmetric. Analogous, we prove that $T$ is $J C J$-symmetric. 
Proposition 3.5. If $T: \mathcal{H} \rightarrow \mathcal{H}$ is both $C$ and J-symmetric, then $T U$ is $C$ symmetric, where $U=C J$.

Proof. In fact, once $T$ is both $C$-symmetric and $J$-symmetric, we have by Lemma 3.4 that $T$ is $C J C$-symmetric and so

$$
(T U) C=T(C J C)=C U^{*} T^{*}=C(T U)^{*} .
$$

Proposition 3.6. An operator $T: \mathcal{H} \rightarrow \mathcal{H}$ is $C$-symmetric if, and only if, $\mathcal{J} T^{*} C=$ $(C \mathcal{J})^{*} T$, where $\mathcal{J}\left(\sum_{n=0}^{\infty} \alpha_{n} f_{n}\right)=\sum_{n=0}^{\infty} \overline{\alpha_{n}} f_{n}$ and $\left\{f_{n}\right\}_{n=0}^{\infty}$ is a orthonormal basis for $\mathcal{H}$.

Proof. We already know that $U=C \mathcal{J}$ is unitary and both $C$ and $\mathcal{J}$-symmetric. Now, note that

$$
\mathcal{J} T^{*} C=(C \mathcal{J})^{*} T \Leftrightarrow U T^{*} C=C U^{*} T .
$$

First see that if $T$ is $C$-symmetric, then $U T^{*} C=U(C T)=\left(C U^{*}\right) T$. Reciprocally, we have

$$
\begin{aligned}
C T^{*} & =C U^{*}\left(U T^{*} C\right) C \\
& =C U^{*}\left(C U^{*} T\right) C \\
& =\left(U C C U^{*}\right) T C \\
& =T C .
\end{aligned}
$$

We end this section with two properties of complex symmetry which we leave the proof to the reader.

Proposition 3.7. Let $U: \mathcal{H} \rightarrow \mathcal{H}$ an unitary operator $J$-symmetric. If $T$ is an operator such that $U T^{*}=T U$ then:

(i) $J T^{*}=T^{*} J \Leftrightarrow T$ is $U J$-symmetric.

(ii) $U J T=T J U^{*} \Leftrightarrow T$ is $J$-symmetric.

\section{Complex symmetry of Aluthge and Duggal transforms}

Recall that the polar decomposition of an operator $T: \mathcal{H} \rightarrow \mathcal{H}$ is uniquely expressed by $T=U|T|$, where $|T|=\sqrt{T^{*} T}$ is a positive operator and $U$ is a partial isometry such that $\operatorname{Ker}(U)=\operatorname{Ker}|U|$ and $U$ maps $\operatorname{cl}(\operatorname{Ran}|T|)$ onto 
$\operatorname{cl}(\operatorname{Ran}(T))$. In this case, the Aluthge and Duggal Transforms are given, respectively, by $\widetilde{T}=|T|^{\frac{1}{2}} U|T|^{\frac{1}{2}}$ and $\widehat{T}=|T| U$.

We already known that the Aluthge transform of a complex symmetric operator is also complex symmetric (see [4, Theorem 1]). In this section we study relations between complex symmetry of $T$ and $|T|$ with relation the conjugations $C$ and $J$, as well as the operators $\widetilde{T}$ and $\widehat{T}$.

Proposition 4.1. If $T$ is complex symmetric, then $|T|$ is also complex symmetric.

Proof. If $C T=T^{*} C$, we have by Remark of [4, Lemma 1] that $T=C J|T|$, where $J$ commutes with $|T|$. Thus, once that $C J$ is a unitary operator, follows that

$$
J|T|=C(C J|T|)=|T|^{*}(C J)^{*} C=|T|^{*} J .
$$

Corollary 4.2. If $T$ is complex symmetric, then $|T|$ is self-adjoint.

Proposition 4.3. Let $C$ and $J$ conjugations on $\mathcal{H}$ such that $T=C J|T|$. If $|T|$ is $C$-symmetric, then $T$ is also $C$-symmetric.

Proof. First, let's show that $|T|$ is $J$-symmetric. In fact, see that

$$
J(J C|T|)=C|T|=|T|^{*} C=\left(|T|^{*} C J\right) J,
$$

and so $J C|T|$ is $J$-symmetric. Thus, by Proposition 3.3, $|T|$ is $J$-symmetric. Therefore, it is enough to see that:

$$
\begin{aligned}
C T & =C(C J|T|) \\
& =|T|^{*} J \\
& =\left(|T|^{*} J C\right) C \\
& =(C J|T|)^{*} C \\
& =T^{*} C .
\end{aligned}
$$

Corollary 4.4. Let $T=C J|T|$. If $|T|$ is $C$-symmetric, then $\widehat{T}=T$.

Corollary 4.5. Let $T=C J|T|$. Then $|T|$ is $C$-symmetric if, and only if, $\widehat{T}$ is J-symmetric. 
Proposition 4.6. Let $T=C J|T|$. If $C|T|=|T|^{*} C$ and $C J=J C$, then $T$ is $J$-symmetric.

Proof. In fact, we have that

$$
\begin{aligned}
J T & =J(C J|T|) \\
& =C|T| \\
& =|T|^{*} J J C \\
& =|T|^{*} J C J \\
& =(C J|T|)^{*} J \\
& =T^{*} J .
\end{aligned}
$$

\section{Bibliography}

[1] A. Brown and P.R. Halmos, Algebraic properties of Toeplitz operators, J.Reine Angew. Math. 213 (1963-1964), 89-102.

[2] R. G. Douglas, Banach Algebra Techniques in Operator Theory, second ed., Graduate Texts in Mathematics, vol. 179, Springer-Verlag, New York, 1998.

[3] M. Fatehi, Complex symmetric weighted composition operators, Complex Variables and Elliptic Equations 64 (2019), 710-720.

[4] S. R. Garcia, Aluthge transforms of complex symmetric operators, Integr. Equ. Oper. Theory. (2008), 1-11.

[5] S.R. Garcia and M. Putinar, Complex symmetric operators and applications, Trans. Amer. Math. Soc. 358 (2006), 1285-1315.

[6] S.R. Garcia and M. Putinar, Complex symmetric operators and applications II, Trans. Amer. Math. Soc. 359 (2007), 3913-3931.

[7] K. Guo and S. Zhu, A canonical decomposition of complex symmetric operators, J. Operator Theory 72 (2014), 529-547.

[8] E. Ko and J. Lee, On complex symmetric Toeplitz operators, J. Math. Anal. Appl. 434 (2016), 20-34.

Received November 11, 2020; revised January 13, 2021; accepted November 21, 2021.

\section{Author information}

Marcos S. Ferreira, Departamento de Ciências Exatas e Tecnológicas, Universidade Estadual de Santa Cruz, Ilhéus, Bahia, Brasil.

E-mail: msferreira@uesc.br 